\title{
Изучение процессов структурообразования керамических покрытий кинетическим методом Монте-Карло
}

\author{
(C) А.В. Черногор, И.В. Блинков, А.П. Демиров \\ Национальный исследовательский технологический университет „МИСиС“, Москва, Россия \\ E-mail: avchernogor@gmail.com
}

Поступило в Редакцию 17 июня 2020 г.

В окончательной редакции 20 июля 2020 г.

Принято к публикации 23 июля 2020 г.

\begin{abstract}
Изучены процессы структурообразования arc-PVD-покрытий $\mathrm{Ti}-\mathrm{Cr}-\mathrm{N}$ методами компьютерного моделирования. Предложен новый подход к определению геометрического пространства для определения положения атомов относительно сформированных димеров и кристаллитов. Рассчитаны параметры структуры и изучены процессы структурообразования покрытий при $120 \mathrm{~V}$.
\end{abstract}

Ключевые слова: моделирование, Монте-Карло, покрытия, структура.

DOI: 10.21883/PJTF.2020.21.50187.18427

В настоящий момент одним из основных направлений развития многих отраслей науки и промышленности является разработка материалов с нанокристаллической структурой. Разработки в области покрытий также в основном связаны с формированием нанокристаллической структуры [1-3]. Однако с учетом большого количества элементов, параметров, а также в связи со сложностью изучения свойств наноструктурных материалов процесс разработки покрытий является трудоемкой задачей. Методы компьютерного моделирования могут существенно сократить не только время поиска, но и расширить понимание физических процессов роста покрытий [4-6].

В настоящей работе предложена модель роста многослойных поликристаллических покрытий $\mathrm{Ti}-\mathrm{Cr}-\mathrm{N}$ на основе кинетического метода Монте-Карло. Данный метод позволяет с высокой скоростью проводить численное интегрирование процессов осаждения с большим количеством переменных. Кроме того, в отличие от других методов, например молекулярной динамики, здесь легко выполнять привязку шага интегрирования к физическому времени [7].

С учетом того, что рост покрытий условно состоит из двух параллельно протекающих процессов (осаждение и диффузия), где состав потока $F$ также зависит от времени, затраченное время $t$ необходимо вычислять исходя из суммарного пути диффузии $R$, пройденного атомами покрытия. При применении кинетического подхода на каждом этапе интеграции все вероятные акты диффузии - переходы $N_{i}$ - группируются по диффузионному барьеру $E_{i}$, после чего рассчитывается общий коэффициент диффузии $R=\sum\left(N_{i} \exp \left(-E_{i} / k T\right)\right)$. Далее выбирается группа переходов $i$ с вероятностью, пропорциональной коэффициенту диффузии этой группы переходов $R_{i}$. Затем с одинаковой вероятностью осуществляется один из указанных переходов, а пройденное время вычисляется как $\Delta t=1 / R_{i}$. На каждом шаге интегрирования процесса диффузии подсчитывает- ся общее пройденное время $t$ суммированием отрезков времени $\Delta t$. Осуществление диффузии выполняется до тех пор, пока сумма всех актов диффузии на данном шаге остается меньше предельно допустимого, которое вычисляется как $N_{d e p} /\left(F_{\max } \mathbf{L}_{y} \mathbf{L}_{x}\right)$, где $N_{d e p}$ - общее количество осажденных атомов, а $\mathbf{L}-$ размерность измерения пространства в указанном направлении, которая составляла $100 \times 100$ атомов.

Вычисление энергии диффузии каждого перехода происходило путем суммирования начальной энергии $E_{0}$, определяемой температурой подложки и потенциалом смещения, и разницы между энергией взаимодействия атома с соседними в новом положении и текущем положении: $E=E_{0}-\Delta E$. Такой подход позволяет реализовать анизотропность диффузии атомов. Для вычисления плазменного потока $\mathrm{Ti}$ и $\mathrm{Cr}$ использовались данные, полученные ранее численным моделированием в [5]. Определение потока атомов азота выполнялось в приближении постоянного равенства соотношения концентраций $c_{\mathrm{Ti}}+c_{\mathrm{Cr}}=c_{\mathrm{N}}$. Начальная энергия атома, только поступившего на подложку, определяется как $E_{0}=Z U+E_{k}$, где $Z-$ средний заряд иона, $U-$ потенциал смещения, $E_{k}$ - кинетическая энергия. По мере совершения актов диффузии начальная энергия атома затухает пропорционально сумме энергий совершенных переходов, но не меньше, чем температура подложки, выраженная в единицах энергии атомов.

Определение возможных переходов является самой сложной частью модели. В самых первых работах пространство представляли в виде клеток, где атом мог занимать строго только центр клетки [4]. В настоящее время такой подход не оправдан ввиду чрезмерной упрощенности, которая приводит к формированию однонаправленных зерен, сливающихся в один большой монолит. Более прогрессивным является представление пространства также в виде клеток, но центр атома не обязательно должен совпадать с центром клетки [8]. 


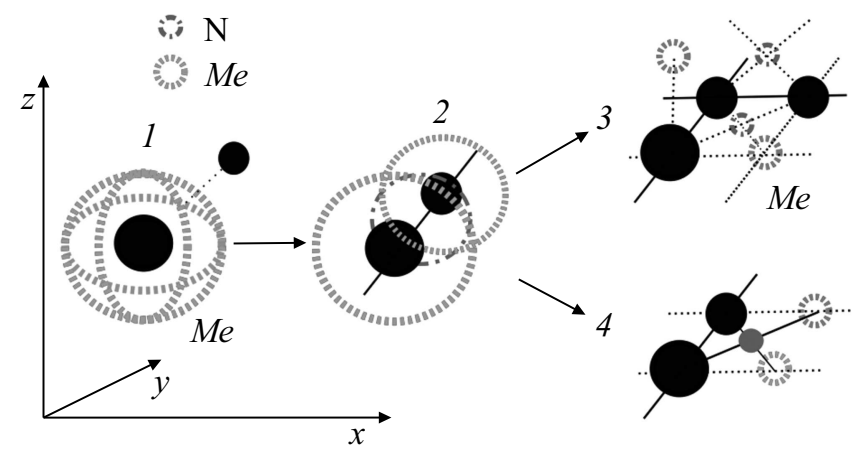

Рис. 1. Модель формирования связей между атомами. Сферой (1) вокруг атома Мe указана область, точку в которой может занять диффундирующий атом $M e$. Окружности, перпендикулярные оси димера (2), соответствуют области, точки в которой могут быть заняты диффундирующим атомом металла или азота. Радиус сферы и окружностей определяется исходя кристаллографических параметров решетки $M e \mathrm{~N}$. Малые окружности (3 и 4) соответствуют точкам в пространстве, в которых может расположиться атом определенного элемента.

В результате возникает новое определение в таких моделях - сайты, т.е. возможные положения атомов, которые определяются исходя из кристаллографических параметров решетки. Однако в конечном итоге в одном из измерений кристаллографические направления остаются жестко зафиксированными.

В настоящей работе предложен новый подход к представлению пространства, часть которого показана на рис. 1. Для свободного атома (1) возможные сайты для диффундирующего атома определяются сферой. Для ди- мера (2) сайты определяются кривыми в пространстве, построенными трансляцией решетки. К сформированному димеру могут присоединиться как атом $\mathrm{Me}$ (3), так и атом $\mathrm{N}$ (4). В итоге формируется кристаллит, для которого мы можем рассчитать ориентацию направлений [100], [010] и [001] в глобальных координатах, а возможные сайты уже будут представлены в виде жестко зафиксированных точек. Как видно, в данном походе пространство не привязано к клеткам, атомы могут занимать любое положение в пространстве, но с учетом выполнения контактного правила. Данное правило ограничивает минимально возможное количество связей с соседними атомами с длиной связи не более параметра решетки $a$. Если в определенном сайте атом не формирует двух связей, то такой сайт считается невозможным и игнорируется для перехода. Таким же образом были проанализированы цепочки присоединения $M e+\mathrm{N}+\mathrm{N}+M e, M e+\mathrm{N}+M e$ и описаны их правила в виде компьютерных алгоритмов. В качестве входных данных по параметрам осаждаемого потока частиц использовались концентрационные профили плазменного потока, представленные в работе [9].

B результате методом arc-PVD были приготовлены покрытия при потенциале смещения $120 \mathrm{~V}$, температуре твердосплавных (ВК6) подложек $450^{\circ} \mathrm{C}$ и давлении азота $0.8 \mathrm{~Pa}$. Все покрытия характеризуются многослойной структурой, которая подробно описана ранее в работе [9]. Поскольку компьютерное моделирование выполнялось только для одного оборота подложек вокруг оси столика, период модуляции соответствует толщине всего покрытия. Период модуляции по компьютерной модели и согласно данным просвечивающей электронной мик-
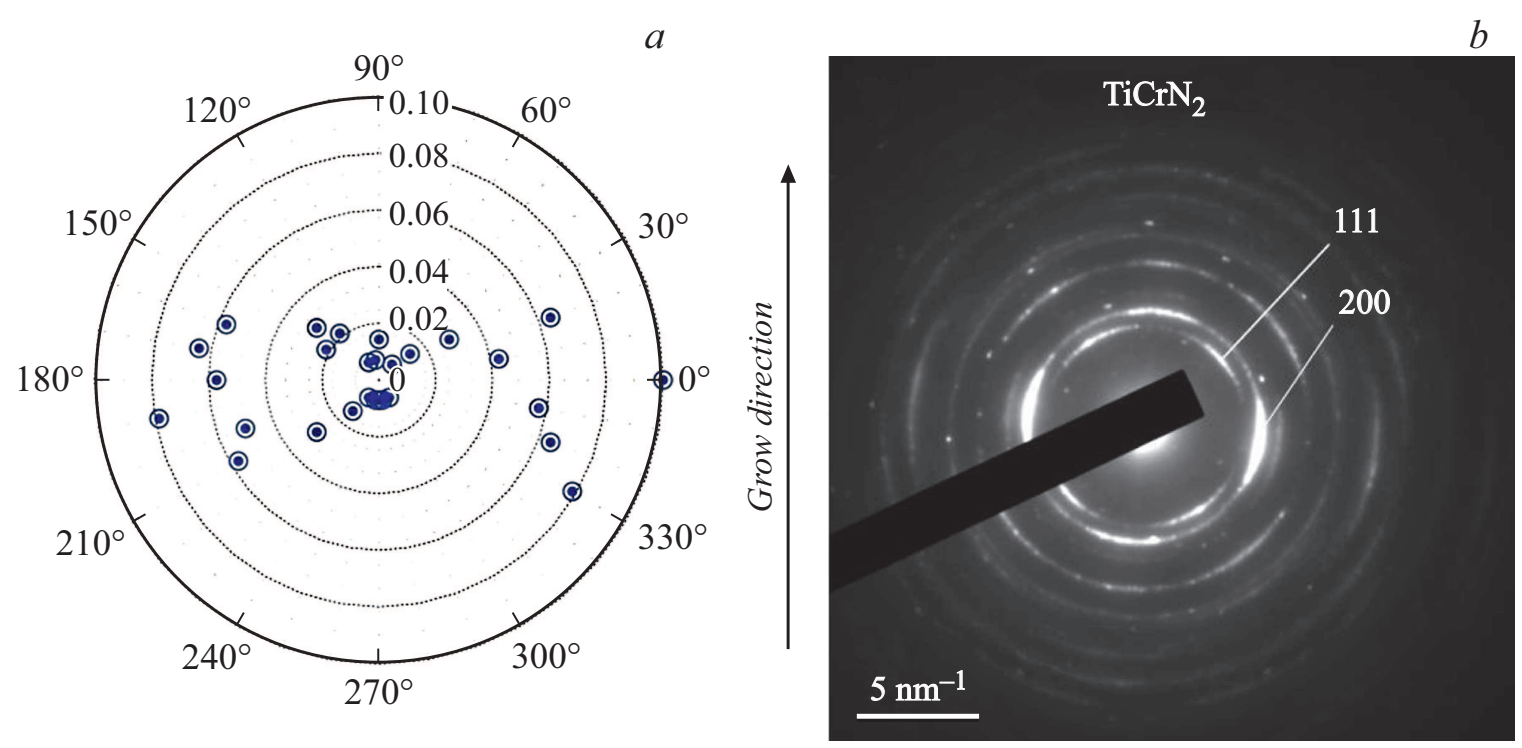

Рис. 2. $a-$ проекция направлений [100] смоделированного покрытия на плоскость, перпендикулярную направлению роста, радиус вектора от точки до центра соответствует относительной доле количества зерен с ориентацией, соответствующей углу поворота. $b$ - электронограмма покрытия, приготовленного при $120 \mathrm{~V}$, полученная с поперечного сечения образца. 


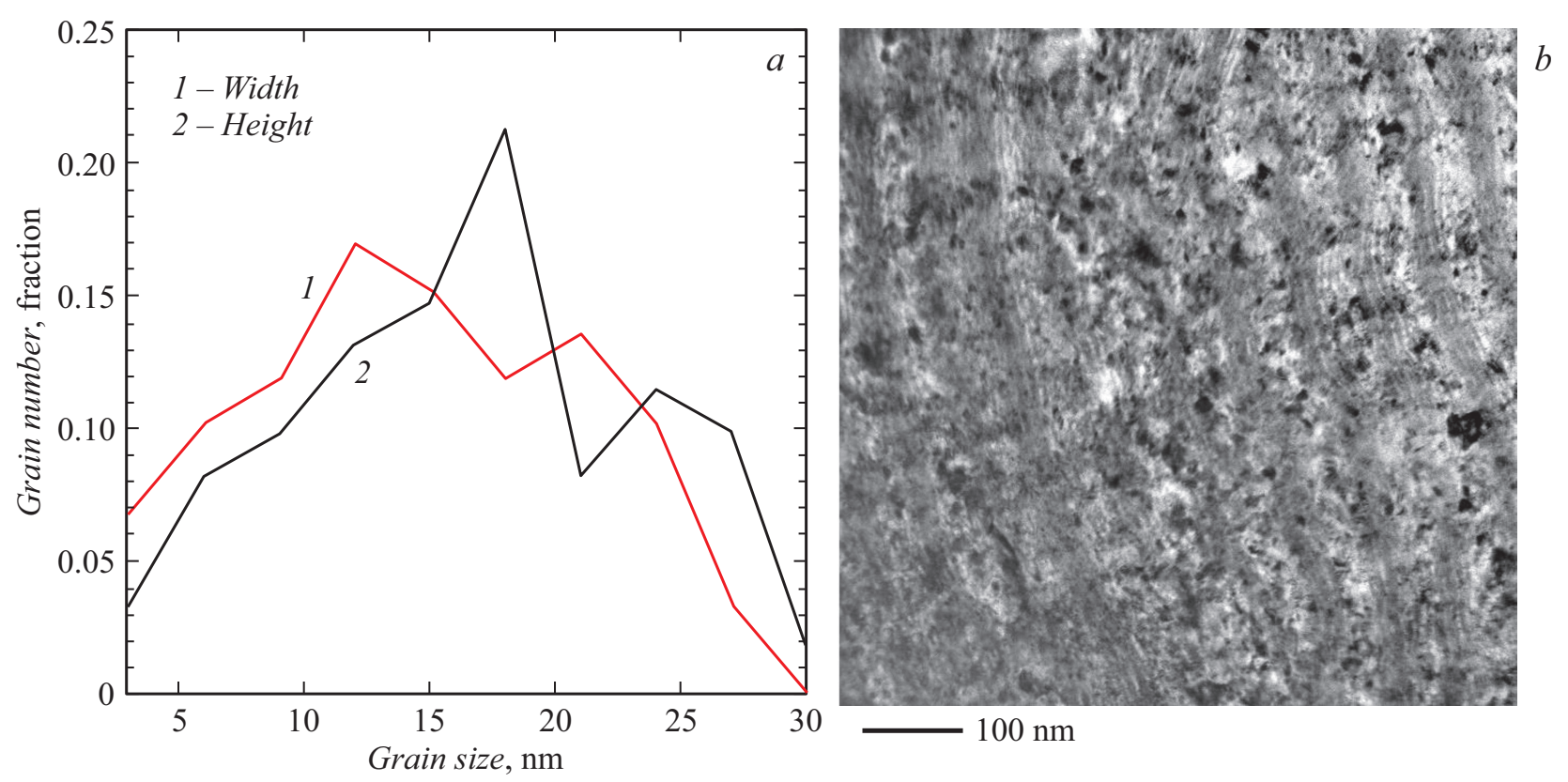

Рис. 3. $a$ - распределение зерен по размеру смоделированного покрытия, высота и ширина зерна определяются исходя из проекции зерен на направления [001] и [100] в глобальных координатах. $b-$ ПЭМ-изображение покрытия в поперечном сечении.

роскопии (ПЭМ) составляет 78 и $52 \mathrm{~nm}$ соответственно. Разница между данными связана с алгоритмом определения энергии связей в соединениях $\mathrm{Ti}-\mathrm{Cr}-\mathrm{N}$. В модели любая связь рассматривалась как нитрид типа $M e \mathrm{~N}$, и это приводило к завышению когезионной энергии. Например, энергия связи в кристалле $M e-M e(-M e)$ будет соответствовать интерметаллиду, а следовательно, разорвать связь будет проще.

Текстура покрытий была проанализирована как проекция кристаллографического направления [100] всех зерен на плоскость, параллельную и перпендикулярную покрытию. Из рис. 2 видно, что большинство зерен ориентировано так, что направление [100] для них перпендикулярно направлению роста покрытий. Такое же поведение наблюдается и на снимках ПЭМ, где дифракция на плоскостях (111) и (200) свидетельствует о том, что зерна повернуты в направлении [100] перпендикулярно росту. Формирование такой текстуры связано с макронапряженным состоянием в покрытиях [10]. Распределение кристаллитов по размеру, представленное на рис. 3, указывает на то, что рост кристаллов происходит преимущественно в плоскости покрытия, что объясняется бомбардировкой с последующим испарением выступающих атомов с поверхности. Такой режим роста способствует формированию плотной беспористой структуры. Размер зерен, полученных экспериментальным путем, больше, и средний размер составляет $27 \pm 6 \mathrm{~nm}$. Однако следует учитывать, что в расчетных данных размер определяется для кристаллитов, тогда как на снимках ПЭМ мы видим зерна. Из-за наложения дифракционных линий от фаз $\mathrm{Ti}-\mathrm{Cr}-\mathrm{N}$ методами рент- геновской дифракции измерить размер кристаллитов не представляется возможным.

Таким образом, кинетический метод Монте-Карло для моделирования структуры позволяет оценивать не только средний размер зерен и распределение элементов в покрытии, но и текстуру. Применение предложенного подхода для описания пространства в целом адекватно описывает размер зерен, их ориентацию в пространстве и толщину покрытия. Отклонение от реальных экспериментов можно уменьшить за счет расширения списка возможных фаз, например в данном случае добавив формирование чистых металлов $\mathrm{Cr}$, Тi и интерметаллидов $\mathrm{Cr}-\mathrm{Ti}$, а также фаз типа $\mathrm{Cr}_{2} \mathrm{~N}$. Единственной сложностью в данном случае является необходимый объем анализа возможных комбинаций формирования димеров и кристаллитов, подобный представленному на рис. 1.

Кроме того, полученный массив данных несет еще больше информации (о таких параметрах, как концентрация вакансий и состав границ раздела слоев), для анализа которой возможно разрабатывать отдельные алгоритмы ее последующей обработки.

\section{Финансирование работы}

Исследование выполнено при финансовой поддержке Российского фонда фундаментальных исследований в рамках научного проекта № 19-38-90113.

\section{Конфликт интересов}

Авторы заявляют, что у них нет конфликта интересов. 


\section{Список литературы}

[1] Машков Ю.К., Коротаев Д.Н., Байбарацкая М.Ю., Алимбаева Б.Ш. // ЖТФ. 2015. Т. 85. В. 10. С. 75-79.

[2] Бессолов В.Н., Компан М.Е., Коненкова Е.В., Пантелеев В.Н., Родин С.Н., Щеглов М.П. // Письма в ЖТФ. 2019. T. 45. B. 11 . C. $3-5$.

DOI: 10.21883/PJTF.2019.11.47813.17756

[3] Bobzin K. // CIRP J. Manufactur. Sci. Technol. 2017. V. 18. P. 1-9. DOI: 10.1016/j.cirpj.2016.11.004

[4] Rodgers W.J., May P.W., Allan N.L., Harvey J.N. // J. Chem. Phys. 2015. V. 142. P. 214707 (1-13).

DOI: $10.1063 / 1.4921540$

[5] Nita F., Mastail C., Abadias G. // Phys. Rev. B. 2016. V. 93. P. 064107 (1-13). DOI: 10.1103/PhysRevB.93.064107

[6] Вольпяс В.А., Тумаркин А.В., Михайлов А.К., Козырев А.Б., Платонов Р.А. // Письма в ЖТФ. 2016. Т. 42. B. 14. C. $87-93$.

[7] Martin-Bragado I., Borges R., Balbuena J.P., Jaraiz M. // Progr. Mater. Sci. 2018. V. 92. P. 1-32. DOI: 10.1016/j.pmatsci.2017.09.003

[8] Bruschi P., Nannini A., Pieri F. // Phys. Rev. B. 2002. V. 63. P. 035406 (1-8). DOI: 10.1103/PhysRevB.63.035406

[9] Черногор А.В., Блинков И.В., Белов Д.С., Сергевнин В.С., Волхонский А.О. // Письма в ЖТФ. 2019. Т. 45. В. 3. C. 16-19. DOI: 10.21883/PJTF.2019.03.47265.17575

[10] Gall D., Kodambaka S., Wall M.A., Petrov I., Greene J.E. // Appl. Phys. Lett. 2003. V. 93. P. 9086-9094.

https://doi.org/10.1063/1.1567797 